\title{
Eberlein decomposition for PV inflation systems
}

\author{
Michael Baake ${ }^{1} \cdot$ Nicolae Strungaru ${ }^{2}$ \\ Received: 14 May 2020 / Revised: 11 March 2021 / Accepted: 7 April 2021 / \\ Published online: 26 April 2021 \\ (C) The Author(s) 2021
}

\begin{abstract}
The Dirac combs of primitive Pisot-Vijayaraghavan (PV) inflations on the real line or, more generally, in $\mathbb{R}^{d}$ are analysed. We construct a mean-orthogonal splitting for such Dirac combs that leads to the classic Eberlein decomposition on the level of the pair correlation measures, and thus to the separation of pure point versus continuous spectral components in the corresponding diffraction measures. This is illustrated with two guiding examples, and an extension to more general systems with randomness is outlined.
\end{abstract}

Keywords Inflation tilings $\cdot$ Diffraction measure $\cdot$ Eberlein decomposition $\cdot$ Spectral components

Mathematics Subject Classification 52C23 · 42A38

\section{Introduction}

Symbolic Pisot-Vijayaraghavan (PV) substitutions induce a much-studied class of dynamical systems under the action of $\mathbb{Z}$. By means of suitable suspensions, they also define natural dynamical systems under the continuous translation action of $\mathbb{R}$. Of particular interest is the self-similar suspension, which turns the symbolic substitution system into a tiling inflation; see [8, Ch. 4] and references therein, as well as $[9,20]$, for general background. This setting is naturally connected with general inflation tilings in $\mathbb{R}^{d}$, which is our point of view here.

As the famous Pisot (or PV) substitution conjecture is still unresolved, despite great effort and progress (see [1] for a summary), it seems a good strategy to consider such systems in a wider setting, where one particularly takes mixed spectra more intofocus.

$凶$ Michael Baake

mbaake@math.uni-bielefeld.de

Nicolae Strungaru

strungarun@macewan.ca

1 Fakultät für Mathematik, Universität Bielefeld, Postfach 100131, 33501 Bielefeld, Germany

2 Department of Mathematical Sciences, MacEwan University, 10700104 Avenue, Edmonton, AB T5J 4S2, Canada 
So, given a general PV inflation system, it is of considerable interest to decompose its spectrum in a constructive fashion. Here, we report on some progress in this direction, where we start from the Dirac comb of a PV inflation and split it into two parts, one of which leads to the pure point part of the diffraction measure and the other to the continuous part. Moreover, this splitting possesses an orthogonality relation in an averaged (or Eberlein) sense, which can also be established for more general systems.

An important predecessor of our approach is the work by Aujogue [2], where an Eberlein-type decomposition is established for measure-theoretic dynamical systems, hence in an almost sure sense. When dealing with the class of primitive inflation tilings (or a characteristic point set representing them), which define strictly ergodic Delone dynamical systems, one wants to achieve such a decomposition constructively, starting from a single member of the dynamical system, that is, from a single point set in $\mathbb{R}^{d}$, say. Below, in view of later extensions, we do not restrict our attention to Delone sets, but allow for more general point sets; see $[32,33,38]$ for some of the theory that will then become useful. Starting from the Dirac comb of such a point set, we construct a splitting into two measures that results in the Eberlein decomposition for the pair correlation measures and do this in such a way that these two measures are mutually orthogonal in an averaged (or Eberlein) sense. For some systems, such a splitting has been used in the treatment of diffraction theory of systems with mixed spectrum, see $[8,38,39]$ and references therein, both for deterministic and for stochastic systems.

The paper is organised as follows. First, in Sect. 2, we recall some of the necessary tools and results, which are systematically formulated for $\mathbb{R}^{d}$ to accommodate selfsimilar inflation tilings in sufficient generality. This is followed by a guiding example for $d=1$ with mixed spectrum, namely a twisted version of the classic Fibonacci tiling, in Sect. 3. Then, we state and prove the central orthogonality result, which takes most of Sect. 4, before we can formulate the decomposition theorem in Sect. 5. Here, we also show how it works for the Thue-Morse system, which has an inflation factor that is not a unit and serves as our second guiding example. Finally, in Sect. 6, we extend our splitting approach to two systems of stochastic nature, namely the interaction-free lattice gas and the Fibonacci random inflation system from [16].

\section{Preliminaries}

Due to our setting with Euclidean inflation tilings, we work with $\mathbb{R}^{d}$. Let us mention in passing that many steps can be generalised to any second countable, locally compact Abelian group as well, but we suppress this in what follows. Let $\mathcal{A}=\left(A_{n}\right)_{n \in \mathbb{N}}$ be an averaging sequence in $\mathbb{R}^{d}$, by which we denote a sequence of compact sets that are nested, meaning $A_{n} \subset A_{n+1}^{\circ}$ for all $n \in \mathbb{N}$, and exhausting, which refers to $\bigcup_{n} A_{n}=\mathbb{R}^{d}$. We call an averaging sequence symmetric when $A_{n}=-A_{n}$ holds for all $n \in \mathbb{N}$ and write this as $\mathcal{A}=-\mathcal{A}$. Later, we shall only consider averaging sequences that have the van Hove property, which is to say that, for any compact set $K \subset \mathbb{R}^{d}$, one has

$$
\lim _{n \rightarrow \infty} \frac{\operatorname{vol}\left(\partial^{K} A_{n}\right)}{\operatorname{vol}\left(A_{n}\right)}=0
$$


where $\left.\partial^{K} C:=\left((C+K) \backslash C^{\circ}\right) \cup\left(\overline{\left(\mathbb{R}^{d} \backslash C\right.}-K\right) \cap C\right)$ for $K$ and $C$ compact; compare [8, p. 29] and references given there for more. Symmetric van Hove averaging sequences in $\mathbb{R}^{d}$ that are widely used include cubes and balls, such as $\left([-n, n]^{d}\right)_{n \in \mathbb{N}}$ or $(\{\|x\| \leq n\})_{n \in \mathbb{N}}$.

Recall that $C_{\mathrm{u}}\left(\mathbb{R}^{d}\right)$ denotes the complex vector space of bounded functions on $\mathbb{R}^{d}$ that are uniformly continuous.

Definition 2.1 Two functions $f, g \in C_{\mathrm{u}}\left(\mathbb{R}^{d}\right)$ are said to possess an Eberlein convolution with respect to, or along, a given averaging sequence $\mathcal{A}$ in $\mathbb{R}^{d}$ if

$$
(f \stackrel{\mathcal{A}}{\circledast} g)(x):=\lim _{n \rightarrow \infty} \frac{1}{\operatorname{vol}\left(A_{n}\right)} \int_{A_{n}} f(x-t) g(t) \mathrm{d} t
$$

exists for all $x \in \mathbb{R}^{d}$. When $\mathcal{A}$ is a fixed averaging sequence that has the van Hove property and is symmetric, so $\mathcal{A}=-\mathcal{A}$, we will usually write $f \circledast g$ instead of $f \stackrel{\mathcal{A}}{\circledast} g$.

At this point, it is relevant to ask whether or when $\circledast$ is commutative.

Lemma 2.2 Let $\mathcal{A}$ be a van Hove averaging sequence in $\mathbb{R}^{d}$, let $f, g \in C_{\mathrm{u}}\left(\mathbb{R}^{d}\right)$, and assume that the Eberlein convolution of $f$ and $g$ exists along $\mathcal{A}$. Then, also the Eberlein convolution of $g$ and $f$ exists, this time along $-\mathcal{A}$, and one has

$$
g \stackrel{-\mathcal{A}}{\circledast} f=f \stackrel{\mathcal{A}}{\circledast} g .
$$

In particular, if $\mathcal{A}$ is also symmetric, one has $f \circledast g=g \circledast f$.

Proof This follows from the following (backwards) calculation,

$$
\begin{aligned}
(f \stackrel{\mathcal{A}}{\circledast} g)(x) & =\lim _{n \rightarrow \infty} \frac{1}{\operatorname{vol}\left(A_{n}\right)} \int_{A_{n}} f(x-s) g(s) \mathrm{d} s \\
& =\lim _{n \rightarrow \infty} \frac{1}{\operatorname{vol}\left(A_{n}\right)} \int_{x-A_{n}} f(r) g(x-r) \mathrm{d} r \\
& =\lim _{n \rightarrow \infty} \frac{1}{\operatorname{vol}\left(A_{n}\right)} \int_{-A_{n}} g(x-r) f(r) \mathrm{d} r=\left(g^{-\mathcal{A}} f\right)(x),
\end{aligned}
$$

where the first equality in the last line is a consequence of the van Hove property of $\mathcal{A}$ together with the boundedness of $f$ and $g$.

From now on, whenever we write $f \circledast g$, it is understood that the Eberlein convolution refers to a symmetric van Hove averaging sequence and is assumed to exist; that is, the averaging limit along $\mathcal{A}$ exists for all $x \in \mathbb{R}^{d}$. Here, we need a generalisation of this notion to translation-bounded Radon measures on $\mathbb{R}^{d}$, which are the measures $\mu$ such that $\sup _{t \in \mathbb{R}^{d}}|\mu|(t+K)<\infty$ for some fixed compact set $K \subset \mathbb{R}^{d}$ with non-empty interior, where $|\mu|$ denotes the total variation of $\mu$. We denote the class of translationbounded Radon measures by $\mathcal{M}^{\infty}\left(\mathbb{R}^{d}\right)$ and say that $\mu, v \in \mathcal{M}^{\infty}\left(\mathbb{R}^{d}\right)$ possess an 
Eberlein convolution with respect to a symmetric van Hove averaging sequence $\mathcal{A}$ if the limit

$$
\mu \circledast v=\lim _{n \rightarrow \infty} \frac{\left.\left.\mu\right|_{A_{n}} * v\right|_{A_{n}}}{\operatorname{vol}\left(A_{n}\right)}
$$

exists in the vague topology, where $\left.\mu\right|_{K}$ denotes the restriction of the measure $\mu$ to a compact set $K \subset \mathbb{R}^{d}$; see [8,31] for background and [24] for further details. Note that this definition is the unique extension of the concept for functions to the setting of Radon measures.

Remark 2.3 The restriction to symmetric van Hove averaging sequences looks a little artificial. It was chosen to bypass a small inconsistency in the standard definition from above, which is widely used in the literature. Indeed, for a general van Hove averaging sequence, a more consistent alternative would be

$$
\mu \stackrel{\mathcal{A}}{\circledast} v:=\lim _{n \rightarrow \infty} \frac{\left.\left.\mu\right|_{-A_{n}} * v\right|_{A_{n}}}{\operatorname{vol}\left(A_{n}\right)},
$$

which satisfies $\mu \stackrel{\mathcal{A}}{\circledast} v=v \stackrel{-\mathcal{A}}{\circledast} \mu$ in analogy to above. This definition is the consistent extension of Definition 2.1. Indeed, when $\mu$ and $\nu$ are absolutely continuous measures with Radon-Nikodym densities $f$ and $g$, respectively, one finds

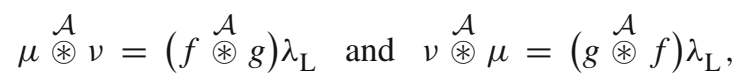

where $\lambda_{\mathrm{L}}$ denotes Lebesgue measure on $\mathbb{R}^{d}$. This observation follows from

$$
\begin{aligned}
\left(\left.\left.\mu\right|_{-A_{n}} * v\right|_{A_{n}}\right)(h) & =\int_{\mathbb{R}^{d}} \int_{\mathbb{R}^{d}} h(x+y) 1_{-A_{n}}(x) f(x) 1_{A_{n}}(y) g(y) \mathrm{d} x \mathrm{~d} y \\
& =\int_{\mathbb{R}^{d}} h(s) \int_{A_{n} \cap\left(s+A_{n}\right)} f(s-y) g(y) \mathrm{d} y \mathrm{~d} s,
\end{aligned}
$$

where $h$ is an arbitrary continuous function with compact support, after dividing by $\operatorname{vol}\left(A_{n}\right)$ and taking the limit as $n \rightarrow \infty$.

When $\mathcal{A}$ is also symmetric, these subtleties go away and $\circledast$ becomes commutative. Since this extra assumption poses no relevant restriction to any of our later arguments, we will usually make it, but always say so in our formal statements.

Next, we need another notion, the Fourier-Bohr (FB) coefficients.

Definition 2.4 A function $g \in C_{\mathrm{u}}\left(\mathbb{R}^{d}\right)$ possesses the $F B$ coefficient at $k \in \mathbb{R}^{d}$ with respect to $\mathcal{A}$ if

$$
c_{g}(k):=\lim _{n \rightarrow \infty} \frac{1}{\operatorname{vol}\left(A_{n}\right)} \int_{A_{n}} \mathrm{e}^{-2 \pi \mathrm{i} k x} g(x) \mathrm{d} x
$$


exists. We say that $g$ possesses FB coefficients relative to $\mathcal{A}$ when $c_{g}(k)$ exists for all $k \in \mathbb{R}^{d}$.

Likewise, a Radon measure $\mu \in \mathcal{M}^{\infty}\left(\mathbb{R}^{d}\right)$ possesses FB coefficients with respect to $\mathcal{A}$ on a set $S \subseteq \mathbb{R}^{d}$ if

$$
c_{\mu}(k):=\lim _{n \rightarrow \infty} \frac{1}{\operatorname{vol}\left(A_{n}\right)} \int_{A_{n}} \mathrm{e}^{-2 \pi \mathrm{i} k x} \mathrm{~d} \mu(x)
$$

exists for all $k \in S$.

From now on, whenever we write $c_{g}(k)$ or $c_{\mu}(k)$, it is understood that the corresponding limit exists.

The following result, which can be derived from [21, Lemma 8] and is a mild variant of [23, Lemma 1.9 and Cor. 1.20], see also [15, Prop. 8.2], provides the connection between the FB coefficients of a translation-bounded Radon measure $\mu$ and the convolutions $\mu * \varphi$ with arbitrary $\varphi \in C_{\mathrm{c}}\left(\mathbb{R}^{d}\right)$, where the latter denotes the space of continuous functions on $\mathbb{R}^{d}$ with compact support. Below, we use $\widehat{\varphi}$ for the Fourier transform of a function $\varphi$, employing the conventions of [8, Ch. 8].

Lemma 2.5 Let $\mathcal{A}$ be a general van Hove averaging sequence in $\mathbb{R}^{d}$, and consider a Radon measure $\mu \in \mathcal{M}^{\infty}\left(\mathbb{R}^{d}\right)$. If $\varphi \in C_{\mathrm{c}}\left(\mathbb{R}^{d}\right)$ and $k \in \mathbb{R}^{d}$, one has

$$
\lim _{n \rightarrow \infty} \frac{1}{\operatorname{vol}\left(A_{n}\right)}\left|\int_{A_{n}} \mathrm{e}^{-2 \pi \mathrm{i} k t}(\varphi * \mu)(t) \mathrm{d} t-\widehat{\varphi}(k) \int_{A_{n}} \mathrm{e}^{-2 \pi \mathrm{i} k t} \mathrm{~d} \mu(t)\right|=0
$$

In particular, if $c_{\mu}(k)$ exists, then so does $c_{\varphi * \mu}(k)$, and one has

$$
c_{\varphi * \mu}(k)=\widehat{\varphi}(k) c_{\mu}(k) .
$$

Conversely, if $c_{\varphi * \mu}(k)$ exists for some $\varphi$ with $\widehat{\varphi}(k) \neq 0$, then $c_{\mu}(k)$ exists as well.

Proof Let us first note that

$$
\begin{aligned}
\widehat{\varphi}(k) \int_{A_{n}} \mathrm{e}^{-2 \pi \mathrm{i} k t} \mathrm{~d} \mu(t) & =\widehat{\varphi}(k) \int_{\mathbb{R}^{d}} 1_{A_{n}}(s) \mathrm{e}^{-2 \pi \mathrm{i} k s} \mathrm{~d} \mu(s) \\
& =\int_{\mathbb{R}^{d}} \int_{\mathbb{R}^{d}} \mathrm{e}^{-2 \pi \mathrm{i} k(r+s)} \varphi(r) \mathrm{d} r 1_{A_{n}}(s) \mathrm{d} \mu(s) \\
& =\int_{\mathbb{R}^{d}} \int_{\mathbb{R}^{d}} 1_{A_{n}}(s) \mathrm{e}^{-2 \pi \mathrm{i} k t} \varphi(t-s) \mathrm{d} \mu(s) \mathrm{d} t,
\end{aligned}
$$

where we used the substitution $t=r+s$ and Fubini's theorem for the last line. 
Consequently, we have

$$
\begin{aligned}
& \left|\int_{A_{n}} \mathrm{e}^{-2 \pi \mathrm{i} k t}(\varphi * \mu)(t) \mathrm{d} t-\widehat{\varphi}(k) \int_{A_{n}} \mathrm{e}^{-2 \pi \mathrm{i} k t} \mathrm{~d} \mu(t)\right| \\
& \quad=\left|\int_{\mathbb{R}^{d}} \int_{\mathbb{R}^{d}} \mathrm{e}^{-2 \pi \mathrm{i} k t}\left(1_{A_{n}}(t)-1_{A_{n}}(s)\right) \varphi(t-s) \mathrm{d} \mu(s) \mathrm{d} t\right| \\
& \quad \leq \int_{\mathbb{R}^{d}} \int_{\mathbb{R}^{d}}\left|1_{A_{n}}(t)-1_{A_{n}}(s)\right||\varphi(t-s)| \mathrm{d}|\mu|(s) \mathrm{d} t .
\end{aligned}
$$

With $K=\operatorname{supp}(\varphi)$, we have $\left|1_{A_{n}}(t)-1_{A_{n}}(s)\right||\varphi(t-s)|=0$ for any $t \notin \partial^{K} A_{n}$, hence

$$
\left|1_{A_{n}}(t)-1_{A_{n}}(s)\right||\varphi(t-s)| \leq 1_{\partial^{K} A_{n}}(t)|\varphi(t-s)|
$$

which then gives

$$
\begin{aligned}
& \frac{1}{\operatorname{vol}\left(A_{n}\right)}\left|\int_{A_{n}} \mathrm{e}^{-2 \pi \mathrm{i} k t}(\varphi * \mu)(t) \mathrm{d} t-\widehat{\varphi}(k) \int_{A_{n}} \mathrm{e}^{-2 \pi \mathrm{i} k t} \mathrm{~d} \mu(t)\right| \\
& \leq \frac{1}{\operatorname{vol}\left(A_{n}\right)} \int_{\mathbb{R}^{d}} \int_{\mathbb{R}^{d}} 1_{\partial^{K} A_{n}}(t)|\varphi(t-s)| \mathrm{d}|\mu|(s) \mathrm{d} t \\
& =\frac{1}{\operatorname{vol}\left(A_{n}\right)} \int_{\partial^{K} A_{n}} \int_{\mathbb{R}^{d}}|\varphi(t-s)| \mathrm{d}|\mu|(s) \mathrm{d} t \\
& =\frac{1}{\operatorname{vol}\left(A_{n}\right)} \int_{\partial^{K} A_{n}}(|\varphi| *|\mu|)(t) \mathrm{d} t \leq\||\varphi| *|\mu|\|_{\infty} \frac{\operatorname{vol}\left(\partial^{K} A_{n}\right)}{\operatorname{vol}\left(A_{n}\right)} .
\end{aligned}
$$

As $n \rightarrow \infty$, the claim now follows from the translation boundedness of $\mu$ and the van Hove property of $\mathcal{A}$ from Eq. (1).

What we wrote down so far are special relations involving continuous characters on $\mathbb{R}^{d}$, which are the elements of the dual group. Since $\mathbb{R}^{d}$ is self-dual, it is common to employ an additive notation for the characters and denote them by $\chi_{k}: \mathbb{R}^{d} \rightarrow \mathbb{S}^{1}$, where $k \in \mathbb{R}^{d}$ is fixed and the mapping is given by

$$
x \mapsto \chi_{k}(x):=\mathrm{e}^{2 \pi \mathrm{i} k x}
$$

Clearly, one then has $\chi_{k}(x) \neq 0$ for all $x \in \mathbb{R}^{d}$, together with $\overline{\chi_{k}}=\chi_{-k}$.

Before we embark on the general result, let us discuss our first guiding example for $d=1$, which is built as a simple extension [5] of the classic Fibonacci tiling of the real line. 


\section{A simple PV inflation with mixed spectrum}

Fix the alphabet $\{a, \underline{a}, b, \underline{b}\}$ and consider the aperiodic substitution rule

$$
\varrho: a \mapsto a b, \quad \underline{a} \mapsto \underline{a} \underline{b}, \quad b \mapsto \underline{a}, \quad \underline{b} \mapsto a,
$$

which has the substitution matrix

$$
M_{\varrho}=\left(\begin{array}{llll}
1 & 0 & 0 & 1 \\
0 & 1 & 1 & 0 \\
1 & 0 & 0 & 0 \\
0 & 1 & 0 & 0
\end{array}\right) .
$$

Since $M_{\varrho}$ has Perron-Frobenius eigenvalue $\tau=\frac{1}{2}(1+\sqrt{5})$, which is a PV unit, with corresponding left eigenvector $(\tau, \tau, 1,1)$, one can turn $\varrho$ into a PV inflation with prototiles (intervals) of length $\tau$ for $a$ and $\underline{a}$, and of length 1 for $b$ and $\underline{b}$; see [5, Sect. 3.2] and references therein for details. On identifying $a$ with $\underline{a}$ and $b$ with $\underline{b}$, one obtains the classic Fibonacci tilings of the real line [8, Ex. 4.6 and Sec. 9.4.1] with pure point spectrum, both in the diffraction and in the dynamical sense. This implies that the 'twisted' inflation system induced by $\varrho$ is an almost everywhere $2: 1$ extension of the classic Fibonacci system. By standard results, see [3] and references therein, this implies that the mapping of the twisted system onto its maximal equicontinuous factor is also 2:1 almost everywhere. Consequently, the twisted system must have mixed spectrum, with a pure point and a continuous part. The latter is purely singular continuous in this case, by [5, Theorem 3.2]. Let us sketch how to arrive at this conclusion constructively.

Now, working with an inflation fixed point and the left endpoints of the intervals of type $\alpha \in\{a, \underline{a}, b, \underline{b}\}$, we always get $\Lambda_{\alpha} \in \mathbb{Z}[\tau]$, wherefore we can employ the natural cut and project scheme (CPS) of the Fibonacci system, abbreviated by $(\mathbb{R}, \mathbb{R}, \mathcal{L}$ ), as constructed and described in detail in [8, Sect. 7.2]. Here, the CPS is given by

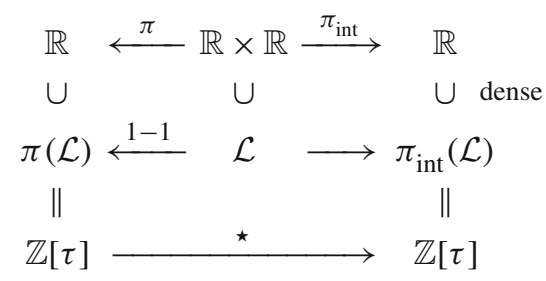

where $\star$ denotes the star map of the CPS, which is the restriction to $\mathbb{Z}(\tau)$ of the unique field automorphism of $\mathbb{Q}(\sqrt{5})$ induced by $\sqrt{5} \mapsto-\sqrt{5}$, and

$$
\mathcal{L}:=\left\{\left(x, x^{\star}\right): x \in \mathbb{Z}[\tau]\right\}
$$

is the Minkowski embedding of $\mathbb{Z}[\tau]$, which is a lattice in $\mathbb{R}^{2} \simeq \mathbb{R} \times \mathbb{R}$ of density $1 / \sqrt{5}$; see [8, Sect. 3.4 and Ex. 7.3] for background and details. Setting $W_{\alpha}=\overline{\Lambda_{\alpha}^{\star}}$, one obtains [5] the compact intervals 


$$
W_{a}=W_{\underline{a}}=[\tau-2, \tau-1] \text { and } W_{b}=W_{\underline{b}}=[-1, \tau-2] .
$$

Now, each set $\curlywedge\left(W_{\alpha}\right):=\left\{x \in \mathbb{Z}[\tau]: x^{\star} \in W_{\alpha}\right\}$ is a regular model set with pure point diffraction [8, Theorem 9.4], while this is not true of the point sets $\Lambda_{\alpha}$.

One can check that each $\Lambda_{\alpha}$, which satisfies $\Lambda_{\alpha} \subseteq \curlywedge\left(W_{\alpha}\right)$ by construction, has half the density of $\curlywedge\left(W_{\alpha}\right)$; compare [8, Sect. 9.4.1] for the results of the two-letter Fibonacci chain. By [10, Theorem 5.3], the point sets $\Lambda_{\alpha}^{\star}$ are uniformly distributed in $W_{\alpha}$, and each of the measures

$$
v_{\alpha}=\delta_{\Lambda_{\alpha}}-\frac{1}{2} \delta_{\curlywedge\left(W_{\alpha}\right)}
$$

has vanishing FB coefficients, where we use $\delta_{S}:=\sum_{x \in S} \delta_{x}$ to denote the Dirac comb of a point set $S$. So, with $\omega_{\alpha}=\frac{1}{2} \delta_{\curlywedge\left(W_{\alpha}\right)}$, one has a decomposition

$$
\delta_{\Lambda_{\alpha}}=\omega_{\alpha}+v_{\alpha}
$$

into two summands. The crucial observation now is that the first gives rise to the pure point part of the spectrum, and the second to the continuous part, while all cross-terms under the Eberlein convolution with respect to any symmetric van Hove averaging sequence in $\mathbb{R}$ vanish. Further details, and a closely related example with a more complicated window structure, are discussed in [10, Sect. 7]. Our goal now is to substantiate the decomposition claim and prove it in sufficient generality.

\section{Orthogonality for Eberlein convolution}

Let us first state an elementary result on the connection between the FB coefficients of a function $f \in C_{\mathrm{u}}\left(\mathbb{R}^{d}\right)$ and the Eberlein convolution of $f$ with the characters on $\mathbb{R}^{d}$.

Lemma 4.1 Let $\mathcal{A}$ be a symmetric van Hove averaging sequence in $\mathbb{R}^{d}$, and consider a function $f \in C_{\mathrm{u}}\left(\mathbb{R}^{d}\right)$ together with a character $\chi_{k}$. Then, with respect to $\mathcal{A}$, the following statements are equivalent.

(1) The FB coefficient $c_{f}(k)$ exists.

(2) The Eberlein convolution $\chi_{k} \circledast f$ exists.

(3) There is some $x \in \mathbb{R}^{d}$ such that $\lim _{n \rightarrow \infty} \frac{1}{\operatorname{vol}\left(A_{n}\right)} \int_{\mathbb{R}^{d}} \chi_{k}(x-t) f(t) \mathrm{d} t$ exists.

Further, assume that $c_{f}(k)$ exists. Then, for all $x \in \mathbb{R}^{d}$, one has the relation

$$
\left(\chi_{k} \circledast f\right)(x)=\chi_{k}(x) c_{f}(k) .
$$

Proof For arbitrary $x \in \mathbb{R}^{d}$ and $n \in \mathbb{N}$, we have

$$
\int_{A_{n}} \chi_{k}(x-t) f(t) \mathrm{d} t=\int_{A_{n}} \chi_{k}(x) \overline{\chi_{k}(t)} f(t) \mathrm{d} t=\chi_{k}(x) \int_{A_{n}} \overline{\chi_{k}(t)} f(t) \mathrm{d} t .
$$


Since $\chi_{k}(x) \neq 0$, the claimed equivalences, as well as the final identity, follow via dividing by $\operatorname{vol}\left(A_{n}\right)$ and taking the limit as $n \rightarrow \infty$.

This has an immediate consequence as follows.

Corollary 4.2 Let $\mathcal{A}$ be a symmetric van Hove averaging sequence in $\mathbb{R}^{d}$, and consider a function $f \in C_{\mathrm{u}}\left(\mathbb{R}^{d}\right)$ together with a trigonometric polynomial $P=\sum_{j=1}^{n} \alpha_{j} \chi_{k_{j}}$. If the FB coefficients $c_{f}\left(k_{j}\right)$ exist for all $1 \leq j \leq n$, the Eberlein convolution $P \circledast f$ exists, too, with

$$
P \circledast f=\sum_{j=1}^{n} \alpha_{j} c_{f}\left(k_{j}\right) \chi_{k_{j}} .
$$

To continue, we need another notion as follows.

Definition 4.3 Let $\mathcal{A}$ be a van Hove averaging sequence in $\mathbb{R}^{d}$ and $\mu \in \mathcal{M}^{\infty}\left(\mathbb{R}^{d}\right)$. If the FB coefficients of $\mu$ with respect to $\mathcal{A}$ exist, the corresponding $F B$ spectrum of $\mu$ is the set $\left\{k \in \mathbb{R}^{d}: c_{\mu}(k) \neq 0\right\}$. Further, we say that $\mu$ has null FB spectrum with respect to $\mathcal{A}$ if, for all $k \in \mathbb{R}^{d}$, one has

$$
c_{\mu}(k)=\lim _{n \rightarrow \infty} \frac{1}{\operatorname{vol}\left(A_{n}\right)} \int_{A_{n}} \overline{\chi_{k}(t)} \mathrm{d} \mu(t)=0,
$$

which is to say that all FB coefficients of $\mu$ exist and vanish.

Likewise, $f \in C_{\mathrm{u}}\left(\mathbb{R}^{d}\right)$ has null FB spectrum with respect to $\mathcal{A}$ if, for all $k \in \mathbb{R}^{d}$, we have

$$
c_{f}(k)=\lim _{n \rightarrow \infty} \frac{1}{\operatorname{vol}\left(A_{n}\right)} \int_{A_{n}} \overline{\chi_{k}(t)} f(t) \mathrm{d} t=0 .
$$

An immediate consequence of Lemma 4.1 and Lemma 2.5 is the following.

Corollary 4.4 Let $\mathcal{A}$ be a symmetric van Hove averaging sequence in $\mathbb{R}^{d}$. Consider a measure $\mu \in \mathcal{M}^{\infty}\left(\mathbb{R}^{d}\right)$ and a function $f \in C_{\mathrm{u}}\left(\mathbb{R}^{d}\right)$. Then, the following two properties hold.

(a) If $\mu$ has null FB spectrum with respect to $\mathcal{A}$, the function $\varphi * \mu$, for any $\varphi \in C_{\mathrm{c}}\left(\mathbb{R}^{d}\right)$, has null FB spectrum with respect to $\mathcal{A}$ as well.

(b) If $f$ has null FB spectrum with respect to $\mathcal{A}$, one has $P \circledast f=0$ for all trigonometric polynomials $P$, where $\circledast$ is the Eberlein convolution along $\mathcal{A}$.

At this point, we can prove the central tool for our later computations, where $\delta_{S}$ is again the Dirac comb of a point set $S \subset \mathbb{R}^{d}$. For generalisations of this result, we refer to [41].

Theorem 4.5 Let $\mathcal{A}$ be a symmetric van Hove averaging sequence in $\mathbb{R}^{d}$, consider a measure $\mu \in \mathcal{M}^{\infty}\left(\mathbb{R}^{d}\right)$, and assume that $\mu$ has null FB spectrum with respect to $\mathcal{A}$. 
Further, let $\Lambda$ be a regular model set in $\mathbb{R}^{d}$. Then, the Eberlein convolution $\mu \circledast \delta_{\Lambda}$ exists along $\mathcal{A}$ and

$$
\mu \circledast \delta_{\Lambda}=0 .
$$

Proof Let $\left(\mathbb{R}^{d}, H, \mathcal{L}\right)$ be the CPS for the description of the model set, where $H$ is a compactly generated LCAG and $\mathcal{L}$ is a lattice in $\mathbb{R}^{d} \times H$, that is, a co-compact discrete subgroup. Note that we need $H$ in this generality because the internal space need not be Euclidean; we refer to [28,29] and [8, Sect. 7.2] for general background, and to $[13,39]$ for a detailed description of the explicit construction of the CPS. Further, let $W \subseteq H$ be the window that gives

$$
\Lambda=\curlywedge(W)=\left\{x \in \pi(\mathcal{L}): x^{\star} \in W\right\}
$$

as a regular model set, where $\pi: \mathbb{R}^{d} \times H \rightarrow \mathbb{R}^{d}$ is the canonical projection and $\star$ denotes the star map of the CPS.

Now, since $\mu$ and $\delta_{\Lambda}$ are translation-bounded measures by construction, the set

$$
\left\{\frac{\left.\left.\mu\right|_{A_{n}} * \delta_{\Lambda}\right|_{A_{n}}}{\operatorname{vol}\left(A_{n}\right)}: n \in \mathbb{N}\right\}
$$

is pre-compact in the vague topology and metrisable [11]. Therefore, to prove that $\mu \circledast \delta_{\Lambda}=0$, it suffices to show that 0 is the only cluster point of this set (or sequence).

Let $\eta$ be any cluster point of this sequence, and let $\mathcal{B}=\left(B_{n}\right)_{n \in \mathbb{N}}$ be a subsequence of $\mathcal{A}$ with respect to which $\eta$ is a limit, which means that $B_{n}=A_{\ell_{n}}$ with $\ell_{n+1}>\ell_{n}$ for all $n \in \mathbb{N}$. Clearly, $\mathcal{B}$ is again a symmetric van Hove averaging sequence, and one has

$$
\eta=\lim _{n \rightarrow \infty} \frac{\left.\left.\mu\right|_{B_{n}} * \delta_{\Lambda}\right|_{B_{n}}}{\operatorname{vol}\left(B_{n}\right)}=\lim _{n \rightarrow \infty} \frac{\left.\mu\right|_{B_{n}} * \delta_{\Lambda}}{\operatorname{vol}\left(B_{n}\right)},
$$

where the second equality follows from the van Hove property of $\mathcal{B}$ via Schlottmann's lemma [36, Lemma 1.2]; see also [11,24].

Now, fix $\varphi, \psi \in C_{\mathrm{c}}\left(\mathbb{R}^{d}\right)$, set $K:=\operatorname{supp}(\varphi)$, and note that the translationboundedness of $\mu$ implies $\|\varphi * \mu\|_{\infty}<\infty$. Let $\varepsilon>0$, and select a function $h \in C_{\mathrm{c}}(H)$ that satisfies $1_{W} \leq h$ together with

$$
\int_{H}\left(h(t)-1_{W}(t)\right) \mathrm{d} t<\frac{\varepsilon}{1+2 \operatorname{dens}(\mathcal{L})\|\varphi * \mu\|_{\infty} \int_{\mathbb{R}^{d}}|\psi(t)| \mathrm{d} t},
$$

which is clearly possible. Further, with $L=\pi(\mathcal{L}) \subset \mathbb{R}^{d}$, let

$$
v=\omega_{h}:=\sum_{x \in L} h\left(x^{\star}\right) \delta_{x}
$$


which is a strongly almost periodic measure, $v \in \mathcal{S A P}\left(\mathbb{R}^{d}\right)$, by [39, Theorem 5.5.2]; see also [22, Theorem 3.1]. Consequently, $\psi * v$ is a Bohr (or uniformly) almost periodic function. This implies that there exists a trigonometric polynomial $P$ in $d$ variables such that

$$
\|\psi * v-P\|_{\infty}<\frac{\varepsilon}{1+2\|\varphi * \mu\|_{\infty}} .
$$

In view of Eq. (2), we now have

$$
\begin{aligned}
(\varphi * \psi * \eta)(0) & =\lim _{n \rightarrow \infty} \frac{1}{\operatorname{vol}\left(B_{n}\right)} \int_{\mathbb{R}^{d}}(\varphi * \psi)(-s) \mathrm{d}\left(\left.\mu\right|_{B_{n}} * \delta_{\Lambda}\right)(s) \\
& =\left.\lim _{n \rightarrow \infty} \frac{1}{\operatorname{vol}\left(B_{n}\right)} \int_{\mathbb{R}^{d}} \int_{\mathbb{R}^{d}}(\varphi * \psi)(-x-y) \mathrm{d} \mu\right|_{B_{n}}(x) \mathrm{d} \delta_{\Lambda}(y) \\
& =\lim _{n \rightarrow \infty} \frac{1}{\operatorname{vol}\left(B_{n}\right)} \int_{\mathbb{R}^{d}} \int_{\mathbb{R}^{d}} \int_{\mathbb{R}^{d}} 1_{B_{n}}(x) \varphi(-x-y-s) \psi(s) \mathrm{d} s \mathrm{~d} \mu(x) \mathrm{d} \delta_{\Lambda}(y),
\end{aligned}
$$

which can be continued via the substitution $r=y+s$ and Fubini's theorem as

$$
\begin{aligned}
(\varphi * \psi * \eta)(0) & =\lim _{n \rightarrow \infty} \frac{1}{\operatorname{vol}\left(B_{n}\right)} \int_{\mathbb{R}^{d}} \int_{\mathbb{R}^{d}} \int_{\mathbb{R}^{d}} 1_{B_{n}}(x) \varphi(-x-r) \psi(r-y) \mathrm{d} r \mathrm{~d} \mu(x) \mathrm{d} \delta_{\Lambda}(y) \\
& =\lim _{n \rightarrow \infty} \frac{1}{\operatorname{vol}\left(B_{n}\right)} \int_{\mathbb{R}^{d}} \int_{\mathbb{R}^{d}} \int_{\mathbb{R}^{d}} 1_{B_{n}}(x) \varphi(-x-r) \psi(r-y) \mathrm{d} \delta_{\Lambda}(y) \mathrm{d} r \mathrm{~d} \mu(x) \\
& =\lim _{n \rightarrow \infty} \frac{1}{\operatorname{vol}\left(B_{n}\right)} \int_{\mathbb{R}^{d}} \int_{\mathbb{R}^{d}} 1_{B_{n}}(x) \varphi(-x-r)\left(\psi * \delta_{\Lambda}\right)(r) \mathrm{d} \mu(x) \mathrm{d} r .
\end{aligned}
$$

Here, we observe that $1_{B_{n}}(x) \varphi(-x-r)=1_{B_{n}}(r) \varphi(-x-r)$ holds for $x \notin \partial{ }^{K} B_{n}$. Due to the van Hove property of $\mathcal{B}$, we have $\lim _{n \rightarrow \infty} \operatorname{vol}\left(\partial^{K} B_{n}\right) / \operatorname{vol}\left(B_{n}\right)=0$, so we get

$$
\begin{aligned}
(\varphi * \psi * \eta)(0) & =\lim _{n \rightarrow \infty} \frac{1}{\operatorname{vol}\left(B_{n}\right)} \int_{\mathbb{R}^{d}} 1_{B_{n}}(r)\left(\psi * \delta_{\Lambda}\right)(r) \int_{\mathbb{R}^{d}} \varphi(-x-r) \mathrm{d} \mu(x) \mathrm{d} r \\
& =\lim _{n \rightarrow \infty} \frac{1}{\operatorname{vol}\left(B_{n}\right)} \int_{\mathbb{R}^{d}} 1_{B_{n}}(r)\left(\psi * \delta_{\Lambda}\right)(r)(\varphi * \mu)(-r) \mathrm{d} r \\
& =\lim _{n \rightarrow \infty} \frac{1}{\operatorname{vol}\left(B_{n}\right)} \int_{B_{n}}\left(\psi * \delta_{\Lambda}\right)(r)(\varphi * \mu)(-r) \mathrm{d} r
\end{aligned}
$$

Therefore, employing a standard $3 \varepsilon$-strategy, we find

$$
\begin{aligned}
|(\varphi * \psi * \eta)(0)| & =\lim _{n \rightarrow \infty} \frac{1}{\operatorname{vol}\left(B_{n}\right)}\left|\int_{B_{n}}\left(\psi * \delta_{\Lambda}\right)(r)(\varphi * \mu)(-r) \mathrm{d} r\right| \\
& \leq \limsup _{n \rightarrow \infty} \frac{1}{\operatorname{vol}\left(B_{n}\right)}\left|\int_{B_{n}}\left(\psi * \delta_{\Lambda}-\psi * v\right)(r)(\varphi * \mu)(-r) \mathrm{d} r\right|
\end{aligned}
$$




$$
\begin{aligned}
& +\limsup _{n \rightarrow \infty} \frac{1}{\operatorname{vol}\left(B_{n}\right)}\left|\int_{B_{n}}(\psi * v-P)(r)(\varphi * \mu)(-r) \mathrm{d} r\right| \\
& +\limsup _{n \rightarrow \infty} \frac{1}{\operatorname{vol}\left(B_{n}\right)}\left|\int_{B_{n}} P(r)(\varphi * \mu)(-r) \mathrm{d} r\right|,
\end{aligned}
$$

where $P$ is the trigonometric polynomial from (4).

Now, we need to estimate the three terms in the last expression of (5), where we begin with the middle one. Here, for all $n \in \mathbb{N}$, we recall (4) and obtain

$$
\frac{1}{\operatorname{vol}\left(B_{n}\right)}\left|\int_{B_{n}}(\psi * v-P)(r)(\varphi * \mu)(-r) \mathrm{d} r\right| \leq\|\psi * v-P\|_{\infty}\|\varphi * \mu\|_{\infty}<\frac{\varepsilon}{2}
$$

which then gives

$$
\limsup _{n \rightarrow \infty} \frac{1}{\operatorname{vol}\left(B_{n}\right)}\left|\int_{B_{n}}(\psi * v-P)(r)(\varphi * \mu)(-r) \mathrm{d} r\right| \leq \frac{\varepsilon}{2}
$$

Next, since $\mu$ has null FB spectrum relative to $\mathcal{A}$ by assumption, hence clearly also relative to the subsequence $\mathcal{B}$, Corollary 4.4 implies

$$
\limsup _{n \rightarrow \infty} \frac{1}{\operatorname{vol}\left(B_{n}\right)}\left|\int_{B_{n}} P(r)(\varphi * \mu)(-r) \mathrm{d} r\right|=|P \stackrel{\mathcal{B}}{\circledast}(\varphi * \mu)|(0)=0 .
$$

The remaining term is a little harder. Here, we have

$$
\begin{aligned}
T_{1} & :=\limsup _{n \rightarrow \infty} \frac{1}{\operatorname{vol}\left(B_{n}\right)}\left|\int_{B_{n}}\left(\psi * \delta_{\Lambda}-\psi * v\right)(r)(\varphi * \mu)(-r) \mathrm{d} r\right| \\
& \leq \limsup _{n \rightarrow \infty} \frac{1}{\operatorname{vol}\left(B_{n}\right)} \int_{B_{n}}\left|\psi * \delta_{\Lambda}-\psi * v\right|(r)\|\varphi * \mu\|_{\infty} \mathrm{d} r \\
& \leq \limsup _{n \rightarrow \infty} \frac{\|\varphi * \mu\|_{\infty}}{\operatorname{vol}\left(B_{n}\right)} \int_{B_{n}}\left(|\psi| *\left|\delta_{\Lambda}-v\right|\right)(r) \mathrm{d} r \\
& =\limsup _{n \rightarrow \infty} \frac{\|\varphi * \mu\|_{\infty}}{\operatorname{vol}\left(B_{n}\right)} \int_{\mathbb{R}^{d}} \int_{\mathbb{R}^{d}} 1_{B_{n}}(r+s) \mathrm{d}\left|\delta_{\Lambda}-v\right|(s)|\psi|(r) \mathrm{d} r \\
& =\limsup _{n \rightarrow \infty} \frac{\|\varphi * \mu\|_{\infty}}{\operatorname{vol}\left(B_{n}\right)} \int_{\mathbb{R}^{d}}\left(v-\delta_{\Lambda}\right)\left(B_{n}-r\right)|\psi|(r) \mathrm{d} r \\
& \leq\|\varphi * \mu\|_{\infty} \int_{\mathbb{R}^{d}}|\psi|(r) \mathrm{d} r \limsup _{n \rightarrow \infty} \frac{\sup _{t \in \mathbb{R}^{d}}\left(v-\delta_{\Lambda}\right)\left(B_{n}-t\right)}{\operatorname{vol}\left(B_{n}\right)},
\end{aligned}
$$

where, in the second-last line, we have used the fact that $v-\delta_{\Lambda}$ is a positive measure. The crucial observation now is that, due to the model set structure with its uniform 
distribution properties [30], the last term satisfies

$$
\limsup _{n \rightarrow \infty} \frac{\sup _{t \in \mathbb{R}^{d}}\left(v-\delta_{\Lambda}\right)\left(B_{n}-t\right)}{\operatorname{vol}\left(B_{n}\right)}=\operatorname{dens}(\mathcal{L}) \int_{H}\left(h(t)-1_{W}(t)\right) \mathrm{d} t
$$

which implies $T_{1}<\frac{\varepsilon}{2}$ via (3) as required.

It now follows from Eq. (5) that $|(\varphi * \psi * \eta)(0)|<\varepsilon$ holds for all $\varepsilon>0$. This implies

$$
\eta((I . \varphi) *(I . \psi))=\eta(I .(\varphi * \psi))=(\varphi * \psi * \eta)(0)=0
$$

where $(I . g)(x):=g(-x)$ for any (continuous) function $g$. Since $\varphi, \psi \in C_{\mathrm{c}}\left(\mathbb{R}^{d}\right)$ were arbitrary, we see that $\eta=0$ holds on the subset

$$
K_{2}\left(\mathbb{R}^{d}\right):=\operatorname{span}\left\{f * g: f, g \in C_{\mathrm{c}}\left(\mathbb{R}^{d}\right)\right\}
$$

which is dense in $C_{\mathrm{c}}\left(\mathbb{R}^{d}\right)$ by a standard approximate identity argument. Consequently, $\eta=0$, and no other cluster point can exist.

Note that Theorem 4.5 remains true if the symmetry assumption on $\mathcal{A}$ is lifted. The proof remains unchanged, except for replacing $B_{n}$ by $-B_{n}$ from Eq. (2) onwards, but commutativity of $\circledast$ is no longer implied; compare Remark 2.3.

\section{Eberlein splitting and decomposition for PV inflations}

Let $\Lambda_{1}, \ldots, \Lambda_{N}$ denote pairwise disjoint point sets in $\mathbb{R}^{d}$ and consider $\Lambda:=\bigcup_{i} \Lambda_{i}$, which we call a typed point set. Let us assume that $\Lambda$ is a Delone set with nice averaging properties, including the types. In particular, given some symmetric van Hove averaging sequence $\mathcal{A}$, we are interested in the situation that the pair correlation measures

$$
\gamma_{i j}:=\widetilde{\delta_{\Lambda_{i}}} \circledast \delta_{\Lambda_{j}}
$$

with respect to $\mathcal{A}$ exist for all $1 \leq i, j \leq N$, where $\tilde{\mu}$ denotes the (possibly complex) Radon measure defined by $\tilde{\mu}(g)=\overline{\mu(\widetilde{g})}$ with $g \in C_{\mathrm{c}}\left(\mathbb{R}^{d}\right)$ and $\widetilde{g}(x)=\overline{g(-x)}$. For instance, this property is guaranteed when the $\Lambda_{i}$ are regular model sets in the same CPS, or when they emerge as the control points of a primitive inflation rule with $N$ prototiles; see $[8,10]$ for background and various details, and [32] for extensions. Below, we mainly consider the case $d=1$, though the setting is general enough to cover higher dimensions as well.

Theorem 5.1 Let $\Lambda=\dot{U}_{1 \leq i \leq N} \Lambda_{i}$ be a typed point set generated from of a primitive $P V$ inflation rule in one dimension that is aperiodic and has a PV unit as inflation factor, and consider the corresponding natural $C P S\left(\mathbb{R}, \mathbb{R}^{m}, \mathcal{L}\right)$ that emerges via the 
classic Minkowski embedding ${ }^{1}$ of the module spanned by the points. Let $W_{i}$ be the attractors of the induced, contractive iterated function system for the windows in internal space, and set

$$
\alpha_{i}:=\frac{\operatorname{dens}\left(\Lambda_{i}\right)}{\operatorname{dens}(\mathcal{L}) \operatorname{vol}\left(W_{i}\right)}, \quad \omega_{i}:=\alpha_{i} \delta_{\curlywedge\left(W_{i}\right)}, \quad \text { and } \quad v_{i}:=\delta_{\Lambda_{i}}-\omega_{i} .
$$

Then, for any symmetric van Hove averaging sequence $\mathcal{A}$ and all $1 \leq i, j \leq N$, one has the splitting $\delta_{\Lambda_{i}}=\omega_{i}+v_{i}$ together with the decomposition and orthogonality relations

$$
\left(\gamma_{i j}\right)_{\mathrm{s}}=\widetilde{\omega}_{i} \circledast \omega_{j}, \quad\left(\gamma_{i j}\right)_{0}=\widetilde{v_{i}} \circledast v_{j}, \quad \text { and } \tilde{\omega_{i}} \circledast v_{j}=\widetilde{v_{i}} \circledast \omega_{j}=0,
$$

where $\gamma_{i j}=\left(\gamma_{i j}\right)_{\mathrm{s}}+\left(\gamma_{i j}\right)_{0}$ is the unique Eberlein decomposition of the pair correlation measures into their strongly almost periodic and their null-weakly almost periodic components.

Proof When the inflation factor is a unit, the induced CPS has a Euclidean internal space; that is, we have $H=\mathbb{R}^{m}$ with $m \geq 1$, where the latter is a consequence of the assumed aperiodicity. This is the situation fully analysed in [10], with dens $\left(\curlywedge\left(W_{i}\right)\right)=$ $\operatorname{dens}(\mathcal{L}) \operatorname{vol}\left(W_{i}\right)$.

Let $\mathcal{A}$ be arbitrary, but fixed. First, by [10, Theorem 5.3], the FB coefficients of $v_{i}$ satisfy

$$
c_{v_{i}}(k)=0
$$

for all $k \in \mathbb{R}$. Then, by Theorem 4.5 , we get $\widetilde{\omega_{i}} \circledast v_{j}=\widetilde{v_{i}} \circledast \omega_{j}=0$ as claimed.

Next, since $\curlywedge\left(W_{i}\right)$ is a regular model set for each $i$, in the same CPS, the Eberlein convolutions $\widetilde{\omega}_{i} \circledast \omega_{j}$ along $\mathcal{A}$ exist and are strongly almost periodic measures, that is,

$$
\widetilde{\omega}_{i} \circledast \omega_{j} \in \mathcal{S} \mathcal{A} \mathcal{P}(\mathbb{R}) .
$$

Likewise, as the $\Lambda_{i}$ emerge from a primitive inflation rule, the pair correlation measures $\gamma_{i j}$ exist and are weakly almost periodic, compare [6,25], so

$$
\gamma_{i j} \in \mathcal{W} \mathcal{A P}(\mathbb{R})
$$

Therefore, as all required limits exist, we obtain the pair correlation measures [6] as

$$
\begin{aligned}
\gamma_{i j} & =\widetilde{\delta_{\Lambda_{i}}} \circledast \delta_{\Lambda_{j}}=\left(\widetilde{\omega_{i}}+\widetilde{v_{i}}\right) \circledast\left(\omega_{j}+v_{j}\right) \\
& =\widetilde{\omega_{i}} \circledast \omega_{j}+\widetilde{\omega_{i}} \circledast v_{j}+\widetilde{v_{i}} \circledast \omega_{j}+\widetilde{v_{i}} \circledast v_{j} \\
& =\widetilde{\omega_{i}} \circledast \omega_{j}+\widetilde{v_{i}} \circledast v_{j},
\end{aligned}
$$

\footnotetext{
1 The underlying construction is explained in full detail in [8, Sect. 3.4].
} 
which implies that every $\widetilde{v_{i}} \circledast v_{j}$ is a weakly almost periodic measure. Moreover, all these measures are Fourier transformable by [6, Lemma 2.1], and we obtain

$$
\widehat{\gamma_{i j}}=\widehat{\widetilde{\omega}_{i} \circledast \omega_{j}}+\widetilde{{\widetilde{v_{i}} \circledast v_{j}}_{j}}
$$

Our system satisfies the consistent phase property, which relates the (generalised) intensities with the Fourier-Bohr coefficients of the system. This connection is also known as the Bombieri-Taylor conjecture and was proved in [21] for a specific class of one-component systems and later generalised in [6] to the primitive inflation systems under consideration here. For measures which are pure point diffractive, the consistent phase property is equivalent to the Besicovitch almost periodicity of the measure [23, Theorem 3.36]. Invoking the results from [10], a simple calculation now gives

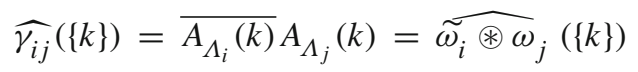

for all $k \in \mathbb{R}$, where the amplitudes satisfy $A_{\Lambda_{j}}(k)=c_{k}\left(\delta_{\Lambda_{j}}\right)$. But this implies

$$
\left({\widetilde{v_{i} \circledast v_{j}}}_{j}\right)_{\mathrm{pp}}=0
$$

which means that $\widetilde{v_{i}} \circledast v_{j} \in \mathcal{W} \mathcal{A} \mathcal{P}_{0}(\mathbb{R})$. Since $\widetilde{\omega}_{i} \circledast \omega_{j} \in \mathcal{S} \mathcal{A} \mathcal{P}(\mathbb{R})$ together with $\widetilde{v_{i}} \circledast v_{j} \in \mathcal{W A P}_{0}(\mathbb{R})$, the claim follows from Eq. (6) and the uniqueness of the Eberlein decomposition [31].

Once again, the result of Theorem 5.1 remains true without the symmetry requirement for $\mathcal{A}$. Indeed, observe that

$$
\widetilde{\widetilde{\omega_{i}} \circledast v_{j}}=\widetilde{v_{j}} \circledast \omega_{i},
$$

which is an easy consequence of $\widetilde{\left.\mu\right|_{K}}=\left.\tilde{\mu}\right|_{-K}$. Then, Theorem 4.5 still provides the two relations we need to derive $(6)$, though $\circledast$ is no longer implied to be commutative.

Remark 5.2 The orthogonality relations in Theorem 5.1, via inserting the definitions of the measures, also imply that

$$
\alpha_{i} \widetilde{\delta_{\curlywedge\left(W_{i}\right)}} \circledast \delta_{\Lambda_{j}}=\alpha_{j} \widetilde{\delta_{\Lambda_{i}}} \circledast \delta_{\curlywedge\left(W_{j}\right)}
$$

holds for all $1 \leq i, j \leq N$, where $\alpha_{i}=\operatorname{dens}\left(\Lambda_{i}\right) / \operatorname{dens}\left(\curlywedge\left(W_{j}\right)\right)$. This proportionality of measures matches nicely with the density formula

$$
\widetilde{\delta_{P} \circledast \delta_{Q}}(\{0\})=\operatorname{dens}(P) \operatorname{dens}(Q)
$$


for Meyer sets $P$ and $Q$ that define uniquely ergodic Delone dynamical systems. With $\mu=\delta_{P}$ and $v=\delta_{Q}$, this relation follows from the complex polarisation identity

$$
\tilde{\mu} \circledast \nu=\frac{1}{4} \sum_{\ell=1}^{4}(-\mathrm{i})^{\ell}\left(\widetilde{\mu+\mathrm{i}^{\ell} v}\right) \circledast\left(\mu+\mathrm{i}^{\ell} v\right),
$$

where the right-hand side is a complex linear combination of four positive-definite measures. Then, under Fourier transform, one gets

$$
\widetilde{\widetilde{\mu} \circledast \nu}(\{0\})=\frac{1}{4} \sum_{\ell=1}^{4}\left|M(\mu)+\mathrm{i}^{\ell} M(\nu)\right|^{2}
$$

from the known intensity formula at 0 , where $M($.$) is the mean of a measure; see [8,$ Prop 9.2] and [31, Lemma 4.10.7]. Since $M(\mu)=\operatorname{dens}(P)$ and $M(v)=\operatorname{dens}(Q)$, Eq. (7) follows by expanding the last sum. It seems interesting to further analyse these identities under the geometric and topological constraints imposed by the projection setting.

The formulation of Theorem 5.1 refers to PV inflations with an inflation multiplier that is a unit, because only this case has been fully treated so far [10]. It is clear that the key result, namely [10, Theorem 5.3], can be generalised to the non-unit situation, and also to PV inflations in higher dimensions. Then, the decomposition remains the same. Let us illustrate the non-unit case with our second guiding example as follows.

Example 5.3 The Thue-Morse (TM) substitution

$$
\varrho: a \mapsto a b, \quad b \mapsto b a
$$

has constant length 2 and gives rise to a partition of the integers, $\Lambda=\mathbb{Z}=\Lambda_{a} \dot{\cup} \Lambda_{b}$. Neither $\Lambda_{a}$ nor $\Lambda_{b}$ is a model set, but our above approach is fully applicable. Here, for the construction of the splitting, we need a CPS with the 2-adic integers as internal space, equipped with its normalised Haar measure that is to be used for the window volumes. Then, the analogue of the construction from Theorem 5.1, for instance, with $\mathcal{A}=([-n, n])_{n \in \mathbb{N}}$, leads to $\omega_{a}=\omega_{b}=\frac{1}{2} \delta_{\mathbb{Z}}$ and thus to the signed measures

$$
v_{a}=\delta_{\Lambda_{a}}-\frac{1}{2} \delta_{\mathbb{Z}} \text { and } v_{b}=-v_{a}
$$

For $\alpha, \beta \in\{a, b\}$, the corresponding pair correlation measures are

$$
\gamma_{\alpha \beta}=\frac{1}{4} \delta_{\mathbb{Z}}+\tilde{v_{\alpha}} \circledast v_{\beta}=\frac{1}{4} \delta_{\mathbb{Z}}+\frac{1}{4} \epsilon_{\alpha \beta} \gamma_{\mathrm{TM}},
$$

with $\epsilon_{\alpha \beta}=1$ or -1 depending on whether $\alpha$ equals $\beta$ or not, and with $\gamma_{\mathrm{TM}}$ denoting the classic autocorrelation measure of the signed TM sequence due to Mahler and Kakutani; see [8, Sect. 10.1] and references therein for details. 
Indeed, (8) is the required Eberlein decomposition. Here, one has $\widehat{\delta_{\mathbb{Z}}}=\delta_{\mathbb{Z}}$ by the Poisson summation formula [8, Prop. 9.4], see also [34], while $\widehat{\gamma_{\mathrm{TM}}}$ is a positive measure that is purely singular continuous. It has the Riesz product representation

$$
\widehat{\gamma_{\mathrm{TM}}}=\prod_{\ell=0}^{\infty}\left(1-\cos \left(2^{\ell+1} \pi(.)\right)\right),
$$

to be interpreted as the vague limit of a sequence of absolutely continuous measures; see [7] for a recent, detailed analysis of this measure.

The explicit extension of this type of analysis to tiling models in higher dimensions, for instance, in the spirit of $[5,6]$, remains an interesting task for the near future, as does a further splitting of the null-weakly almost periodic part, as in [40], according to the different continuous types after Fourier transform.

\section{Further directions}

It is natural to ask whether our constructive approach can be made to work also beyond the PV inflation case and thus perhaps add extra insight into systems that are covered by [2]. In particular, it is of interest to see the orthogonality in the Eberlein sense in more generality, at least almost surely with respect to some given invariant measure in a dynamical systems context. This is indeed possible, and we outline this with two concrete cases.

\subsection{Eberlein splitting for a lattice gas}

Let $0<p<1$ be fixed, and consider the Bernoulli lattice gas on $\mathbb{Z}$ with independent occupation probability $p$ for the single sites. A realisation of this process can thus either be seen as a configuration, meaning an element of $\{0,1\}^{\mathbb{Z}}$, or as a subset $\Lambda \subseteq \mathbb{Z}$. Let us take the latter view, and select a typical subset $\Lambda$, thus with density $p$. By [8, Ex. 11.2], the corresponding autocorrelation measure is

$$
\gamma=p^{2} \delta_{\mathbb{Z}}+p(1-p) \delta_{0}=(\gamma)_{\mathrm{s}}+(\gamma)_{0}
$$

which applies to almost all realisations of the Bernoulli process. This can easily be proved with the strong law of large numbers, see [12] for a detailed exposition, and provides the Eberlein decomposition of $\gamma$ into its strongly almost periodic and its null-weakly almost periodic part. Indeed, the diffraction measure then simply reads $\widehat{\gamma}=p^{2} \delta_{\mathbb{Z}}+p(1-p) \lambda_{\mathrm{L}}$, where $\lambda_{\mathrm{L}}$ denotes Lebesgue measure and $\widehat{\gamma}$ applies to almost all realisations.

Now, in analogy to our above splitting, we set

$$
\delta_{\Lambda}=\omega+v \quad \text { with } \omega=p \delta_{\mathbb{Z}} \text { and } v=\delta_{\Lambda}-\omega
$$


Using $\mathcal{A}=([-n, n])_{n \in \mathbb{N}}$ as before, we get $\delta_{\mathbb{Z}} \circledast \delta_{\mathbb{Z}}=\delta_{\mathbb{Z}}$ from $[8$, Ex. 8.10], hence

$$
\omega \circledast \widetilde{\omega}=\omega \circledast \omega=p^{2} \delta_{\mathbb{Z}}=(\gamma)_{\mathrm{s}}
$$

and $\omega \circledast \delta_{\mathbb{Z}}=p \delta_{\mathbb{Z}}$. Furthermore, $\delta_{\Lambda} \circledast \delta_{\mathbb{Z}}=\delta_{-\Lambda} \circledast \delta_{\mathbb{Z}}=p \delta_{\mathbb{Z}}$ follows from a simple density calculation, where one observes that $-\Lambda$ is another typical realisation if $\Lambda$ is one. But this implies the orthogonality relations $\omega \circledast \widetilde{v}=\widetilde{\omega} \circledast \nu=0$ together with

$$
v \circledast \widetilde{v}=\delta_{\Lambda} \circledast \delta_{-\Lambda}-p^{2} \delta_{\mathbb{Z}}=\gamma-p^{2} \delta_{\mathbb{Z}}=p(1-p) \delta_{0}=(\gamma)_{0} .
$$

We thus see that (9) provides the splitting of $\delta_{\Lambda}$ in complete analogy to Theorem 5.1 and applies to almost all realisations of the lattice gas.

Clearly, this can be extended to higher dimensions, for instance, via replacing $\mathbb{Z}$ by $\mathbb{Z}^{d}$ in the above example, and to many other stochastic systems, as treated in [8, Ch. 11] or in [4]. The constructive splitting approach gives a slightly different interpretation to the method put forward in [4,26], where the splitting is done on the autocorrelation level by separating the mean from the fluctuations. This certainly deserves further clarification in the setting of the Bartlett spectrum from the theory of stochastic processes.

\subsection{Eberlein splitting for a random inflation}

Here, we return to the classic Fibonacci inflation that underlies Sect. 3 and turn it into a random inflation by setting

$$
\varrho: b \mapsto a, \quad a \mapsto \begin{cases}a b, & \text { with probability } p, \\ b a, & \text { with probability } 1-p,\end{cases}
$$

where $p \in[0,1]$ is fixed, and the rule is applied locally. This defines a system that was first analysed in [16] and has recently turned into an interesting paradigm for a random system with both long-range order and some form of disorder $[18,19,35]$. Here, we only consider the geometric setting where $a$ and $b$ stand for intervals of length $\tau$ and 1 , respectively, with normalised Dirac measures on their left endpoints.

From [14, Theorem 3.19], we know that the diffraction measure almost surely satisfies

$$
\widehat{\gamma}=(\widehat{\gamma})_{\mathrm{pp}}+(\widehat{\gamma})_{\mathrm{ac}}
$$

where explicit expressions can be given. Clearly, $(\widehat{\gamma})_{\mathrm{ac}}=0$ for $p=0$ or $p=1$. To set this into our above scheme, let $\Lambda=\Lambda_{a} \dot{\cup} \Lambda_{b}$ be the (typed) control points of a typical realisation of the random Fibonacci inflation, say with $0<p<1$ to avoid the deterministic limiting cases. Then, as shown in [14], there are weighted Dirac combs 
$\omega_{\alpha}$, with $\alpha \in\{a, b\}$, of the form

$$
\omega_{\alpha}=\sum_{x \in \mathbb{Z}[\tau]} h_{\alpha}\left(x^{\star}\right) \delta_{x}
$$

with functions $h_{\alpha}$ that are continuous and supported on the window $[-\tau, \tau]$ of the covering model set. In particular, these Dirac combs are discretely supported in the regular model set $\curlywedge([-\tau, \tau])$, and both have pure point spectrum [39]. What is more, as follows from [14], the pure point part of the diffraction of $\Lambda_{\alpha}$ agrees with the diffraction of $\omega_{\alpha}$.

Thus, using $\omega_{\alpha}$ and defining $v_{\alpha}=\delta_{\Lambda_{\alpha}}-\omega_{\alpha}$, where the latter is a random measure for each $\alpha \in\{a, b\}$, we almost surely (in the sense of the underlying process) are in a situation analogous to the one from Theorem 5.1. In particular, for fixed $u_{a}, u_{b} \in \mathbb{C}$, the random weighted Dirac comb

$$
\mu=u_{a} \delta_{\Lambda_{a}}+u_{b} \delta_{\Lambda_{b}}
$$

almost surely has the autocorrelation $\gamma=(\gamma)_{\mathrm{s}}+(\gamma)_{0}$ where $(\gamma)_{\mathrm{s}}$ agrees with the autocorrelation of $u_{a} \omega_{a}+u_{b} \omega_{b}$ and $(\gamma)_{0}$ is the autocorrelation of $u_{a} v_{a}+u_{b} v_{b}$. Taking Fourier transforms brings us back to (10), where we refer to [14,27,37] for explicit formulas. Once again, this gives a constructive variant of the decomposition advocated in [2], which is fully compatible with the statistical separation of mean and variance from $[4,26]$. In fact, the latter approach quite generally seems to lead to a related further decomposition into singular versus absolutely continuous components, at least for the class of random inflations [17], which opens a promising path to future investigations.

Acknowledgements We thank Philipp Gohlke, Uwe Grimm, Neil Mañibo and Timo Spindeler for helpful discussions and comments on the manuscript. We thank two referees for their thoughtful comments, which helped to improve the presentation. This work was supported by the German Research Foundation (DFG), within the CRC 1283 at Bielefeld University (MB), and by the Natural Sciences and Engineering Council of Canada (NSERC), via grant 2020-00038 (NS).

Funding Open Access funding enabled and organized by Projekt DEAL.

Open Access This article is licensed under a Creative Commons Attribution 4.0 International License, which permits use, sharing, adaptation, distribution and reproduction in any medium or format, as long as you give appropriate credit to the original author(s) and the source, provide a link to the Creative Commons licence, and indicate if changes were made. The images or other third party material in this article are included in the article's Creative Commons licence, unless indicated otherwise in a credit line to the material. If material is not included in the article's Creative Commons licence and your intended use is not permitted by statutory regulation or exceeds the permitted use, you will need to obtain permission directly from the copyright holder. To view a copy of this licence, visit http://creativecommons.org/licenses/by/4.0/.

\section{References}

1. Akiyama, S., Barge, M., Berthé, V., Lee, J.-Y., Siegel, A.: On the Pisot substitution conjecture, in [20], pp. 33-72

2. Aujogue, J.-B.: Pure point/continuous decomposition of translation-bounded measures and diffraction. Ergod. Theory Dyn. Syst. 40, 309-352 (2020). arXiv:1510.06381 
3. Aujogue, J.-B., Barge, M., Kellendonk, J., Lenz, D.: Equicontinuous factors, proximality and Ellis semigroup for Delone sets, in [20], pp. 137-194. arXiv:1407.1787

4. Baake, M., Birkner, M., Moody, R.V.: Diffraction of stochastic point sets: Explicitly computable examples. Commun. Math. Phys. 293, 611-660 (2010). arXiv:0803.1266

5. Baake, M., Frank, N.P., Grimm, U.: Three variations on a theme by Fibonacci. Stoch. Dyn. 21, 2140001:1-23 (2021). arXiv:1910.00988

6. Baake, M., Gähler, F., Mañibo, N.: Renormalisation of pair correlation measures for primitive inflation rules and absence of absolutely continuous diffraction. Commun. Math. Phys. 370, 591-635 (2019). arXiv: 1805.09650

7. Baake, M., Gohlke, P., Kesseböhmer, M., Schindler, T.: Scaling properties of the Thue-Morse measure. Discrete Contin. Dyn. Syst. A 39, 4157-4185 (2019). arXiv:1810.06949

8. Baake, M., Grimm, U.: Aperiodic Order. Vol. 1: A Mathematical Invitation. Cambridge University Press, Cambridge (2013)

9. Baake, M., Grimm, U. (eds.): Aperiodic Order. Vol. 2: Crystallography and Almost Periodicity. Cambridge University Press, Cambridge (2017)

10. Baake, M., Grimm, U.: Fourier transform of Rauzy fractals and point spectrum of 1D Pisot inflation tilings. Doc. Math. 25, 2303-2337 (2020). arXiv:1907.11012

11. Baake, M., Lenz, D.: Dynamical systems on translation bounded measures: Pure point dynamical and diffraction spectra. Ergod. Theory Dyn. Syst. 24, 1867-1893 (2004). arXiv:math.DS/0302231

12. Baake, M., Moody, R.V.: Diffractive point sets with entropy. J. Phys. A Math. Gen. 31, 9023-9039 (1998). arXiv:math-ph/9809002

13. Baake, M., Moody, R.V.: Weighted Dirac combs with pure point diffraction. J. Reine Angew. Math. (Crelle) 573, 61-94 (2004). arXiv:math.MG/0203030

14. Baake, M., Spindeler, T., Strungaru, N.: Diffraction of compatible random substitutions in one dimension. Indag. Math. 29, 1031-1071 (2018). arXiv:1712.00323

15. Gil de Lamadrid, J., Argabright, L.N.: Almost periodic measures. Memoirs Am. Math. Soc. Vol. 85(428), AMS, Providence, RI (1990)

16. Godrèche, C., Luck, J.-M.: Quasiperiodicity and randomness in the plane. J. Stat. Phys. 55, 1-28 (1987)

17. Gohlke, P.: Private communication (2021)

18. Gohlke, P., Rust, D., Spindeler, T.: Shifts of finite type and random substitutions. Discrete Contin. Dyn. Syst. A 39, 5085-5103 (2019). arXiv:1712.05340

19. Gohlke, P., Spindeler, T.: Ergodic frequency measures for random substitutions. Studia Math. 255, 265-301 (2020). arXiv:1810.11122

20. Kellendonk, J., Lenz, D., Savinien, J. (eds.): The Mathematics of Aperiodic Order. Birkhäuser, Basel (2015)

21. Lenz, D.: Continuity of eigenfunctions of uniquely ergodic dynamical systems and intensity of Bragg peaks. Commun. Math. Phys. 287, 225-258 (2009). arXiv:math-ph/0608026

22. Lenz, D., Richard, C.: Pure point diffraction and cut and project schemes for measures: The smooth case. Math. Z. 256, 347-378 (2007). arXiv:math.DS/0603453

23. Lenz, D., Spindeler, T., Strungaru, N.: Pure point diffraction and mean, Besicovitch and Weyl almost periodicity, preprint (2020). arXiv:2006.10821

24. Lenz, D., Spindeler, T., Strungaru, N.: Pure point spectrum for dynamical systems and mean almost periodicity, preprint (2020). arXiv:2006.10825

25. Lenz, D., Strungaru, N.: On weakly almost periodic measures. Trans. Amer. Math. Soc. 371, 6843-6881 (2019). arXiv:1609.08219

26. Luck, J.-M.: A classification of critical phenomena on quasi-crystals and other aperiodic structures. Europhys. Lett. 24, 359-364 (1993)

27. Moll, M.: Diffraction of random noble means words. J. Stat. Phys. 156, 1221-1236 (2014). arXiv:1404.7411

28. Moody, R.V.: Meyer sets and their duals. In: Moody, R.V. (ed.) The Mathematics of Long-Range Aperiodic Order. NATO ASI Ser. C 489, pp. 403-441. Kluwer, Dordrecht (1997)

29. Moody, R.V.: Model sets: a survey. In: Axel, F., Dénoyer, F., Gazeau, J.P. (eds.) From Quasicrystals to More Complex Systems, pp. 145-166. EDP Sciences, Les Ulis, and Springer, Berlin (2000). arXiv:math.MG/0002020

30. Moody, R.V.: Uniform distribution in model sets. Can. Math. Bull. 45, 123-130 (2002)

31. Moody, R.V., Strungaru, N.: Almost periodic measures and their Fourier transforms, in [9], pp. 173-270 
32. Müller, P., Richard, C.: Ergodic properties of randomly coloured point sets. Can. J. Math. 65, 349-402 (2013). arXiv: 1005.4884

33. Richard, C.: Dense Dirac combs in Euclidean space with pure point diffraction. J. Math. Phys. 44, 4436-4449 (2003). arXiv:math-ph/0302049

34. Richard, C., Strungaru, N.: Pure point diffraction and Poisson summation. Ann. H. Poincaré 18, 39033931 (2017). arXiv:1512.00912

35. Rust, D., Spindeler, T.: Dynamical systems arising from random substitutions. Indag. Math. 29, 11311155 (2018). arXiv: 1707.09836

36. Schlottmann, M.: Generalised model sets and dynamical systems. In: Baake, M., Moody, R.V. (eds.) Directions in Mathematical Quasicrystals. CRM Monograph Series, vol. 13, pp. 143-159. AMS, Providence, RI (2000)

37. Spindeler, T.: Spectral Theory of Compatible Random Inflation Systems, $\mathrm{PhD}$ thesis, Univ. Bielefeld (2018). urn:nbn:de:0070-pub-29173839

38. Strungaru, N.: On weighted Dirac combs supported inside model sets. J. Phys. A Math. Theor. 47(335202), 1-19 (2014). arXiv:1309.7947

39. Strungaru, N.: Almost periodic pure point measures, in [9], pp. 271-342. arXiv:1501.00945

40. Strungaru, N.: On the Fourier analysis of measures with Meyer set support. J. Funct. Anal. 278, 108404:1-30 (2020). arXiv:1807.03815

41. Strungaru, N.: On the orthogonality of measures of different spectral type with respect to Eberlein convolution, in preparation

Publisher's Note Springer Nature remains neutral with regard to jurisdictional claims in published maps and institutional affiliations. 